\title{
Intraepithelial Melanocytic Hyperplasia
}

National Cancer Institute

\section{Source}

National Cancer Institute. Intraepithelial Melanocytic Hyperplasia. NCI Thesaurus. Code C35840.

A hyperplasia of the intraepithelial melanocytes. 